\title{
TITLE:
}

\section{Charmonium mass in hot and dense hadronic matter}

$\operatorname{AUTHOR}(S)$ :

Morita, Kenji; Lee, Su Houng

\section{CITATION:}

Morita, Kenji ... [et al]. Charmonium mass in hot and dense hadronic matter. Physical Review C 2012, 85(4): 044917.

ISSUE DATE:

2012-04

URL:

http://hdl.handle.net/2433/155799

RIGHT:

(C2012 American Physical Society 
PHYSICAL REVIEW C 85, 044917 (2012)

\title{
Charmonium mass in hot and dense hadronic matter
}

\author{
Kenji Morita ${ }^{1,2, *}$ and Su Houng Lee ${ }^{3, \dagger}$ \\ ${ }^{1}$ GSI, Helmholzzentrum für Schwerionenforschung, Planckstrasse 1, D-64291 Darmstadt, Germany \\ ${ }^{2}$ Yukawa Institute for Theoretical Physics, Kyoto University, Kyoto 606-8502, Japan \\ ${ }^{3}$ Institute of Physics and Applied Physics, Yonsei University, Seoul 120-749, Korea
}

(Received 17 November 2011; published 17 April 2012)

\begin{abstract}
We investigate mass shifts of charmonia driven by change of the gluon condensate below but near transition temperatures at finite baryonic chemical potential. Extending previous prescription on the relation between gluon condensates and thermodynamic quantities, we model the gluon condensates of hadronic matter at finite temperature and baryonic chemical potential such that the scalar gluon condensate fits with the latest lattice QCD data. By making use of the QCD sum rule and the second-order Stark effect, we find that the smoother transition in the full QCD can lead to moderate mass shifts of charmonia even below the transition temperature. We also find larger mass shift at fixed temperature as chemical potential increases. Existing data on charmonium-charmonium ratio is found to be consistent with the statistical hadronization scenario, including the obtained mass shift.
\end{abstract}

DOI: 10.1103/PhysRevC.85.044917

PACS number(s): 24.85.+p, 11.55.Hx, 14.40.Pq

\section{INTRODUCTION}

Properties of heavy quarkonia in medium have been extensively studied since it was pointed out that suppression of $J / \psi$ by Debye screening could be a signature of creation of the deconfined matter in relativistic heavy ion collisions [1]. It should be noted that a mass shift of a charmonium state in hot hadronic environment could be a precursor phenomenon of the transition caused by a decrease in the string tension [2]. These early expectations are based on an intuitive picture on a quarkonium, a heavy quark and its antiquark bound by a confining potential, which successfully describes the properties in vacuum [3]. While the lattice $\mathrm{QCD}$ provides a first principle approach to the problem, it still lacks the necessary resolution needed for discriminating possible changes in the quarkonium spectral function at finite temperature, especially near the critical temperature where an abrupt change could take place. The maximum entropy method for this problem can at best only tell us about the (non)existence of the lowest peak in the spectral function [4-8]. Therefore, to assess the medium modification, one needs a complementary framework such as the potential model, which utilizes a quark-antiquark potential extracted from lattice calculation [9]. In the meantime, we have proposed an approach utilizing the QCD sum rule and the second-order Stark effect, which allows us to relate the temperature-dependent gluon condensates as the primary inputs from lattice QCD to the spectral changes of heavy quarkonia [10-14].

So far, our studies relied on the gluon condensates extracted from the trace anomaly of pure $\mathrm{SU}(3)$ case [15]. In pure gauge theory with $N_{c} \geqslant 3$, there is a first-order deconfinement transition at $T=T_{c}$; thus, the trace anomaly shows abrupt change across $T_{c}$ [16], which leads to the similar behavior of the spectral property of the quarkonia.

\footnotetext{
*kmorita@yukawa.kyoto-u.ac.jp

†suhoung@yonsei.ac.kr
}

In order to compare results with experimental data, we need more realistic estimates of the condensates based on full QCD lattice calculations, including dynamical light quarks. Recently, the calculation of the equation of state has been carried out with physical quark masses at vanishing chemical potential [17]. Due to the crossover nature of the transition [18], the critical temperature is no longer a well-defined quantity. The pseudocritical temperature defined by a peak or an inflection point depends on observables [19] and is found to range from 147 (chiral susceptibility) to $165 \mathrm{MeV}$ (strange quark number susceptibility).

As for the scalar gluon condensate, which is the gluonic part of the trace anomaly, it is expected to have the same bulk property but with smoother change near the pseudocritical temperature $T_{p c}$. In fact, the magnitude for the change of the scalar condensate in a pure gauge theory at the transition region has been found to be almost the same as in the full QCD case $[11,20]$, when the temperatures are normalized by $T_{c}$ and $T_{p c}$, despite the difference in the (pseudo)critical temperatures; $T_{c} \simeq 265 \mathrm{MeV}$ in the pure $\mathrm{SU}(3)[15,16]$ and $T_{p c} \simeq 190-$ $200 \mathrm{MeV}$ in full (2+1) flavor QCD with $m_{\pi} \simeq 220 \mathrm{MeV}$ [20]. The crossover nature of the transition has led to the smoother temperature dependence of the gluon condensate near $T_{p c}$ [21]. For the twist-2 gluon condensate, which is not a dominant but non-negligible contribution to the sum rule $[11,22]$, no lattice data at physical quark masses is available yet.

In this paper, we first extract the gluon condensates in full QCD by making use of the resonance gas model. This prescription enables us to study a finite baryonic chemical potential case also. Using these gluon condensates, we investigate the change of spectral properties of charmonia in hot hadronic matter at various values of temperature and baryonic chemical potential along the freeze-out line, which is deduced from statistical model analyses $[23,24]$ and is expected to be close to the hadronization points. Although the charmonium production mechanism in relativistic heavy ion collisions has not been understood well, some experimental data seem to indicate that the statistical production of charmonia could be possible [25]. 
Despite the complexity of the collision processes, this scenario considerably simplifies the charmonium-charmonium particle number ratio. We examine possible influences of the spectral modification on this quantity.

In the next section, we present a resonance gas model for the gluon condensates of hot and dense hadronic matter. In Sec. III, we report results of spectral changes of charmonia. The experimental implication will be discussed in Sec. IV. Section V is devoted to a summary.

\section{RESONANCE GAS MODEL FOR THE GLUON CONDENSATES}

We start with two quantities, $M_{0}$ and $M_{2}$, characterizing the temperature dependence of the thermal expectation value of gluonic operators:

$$
\begin{gathered}
\left\langle\frac{\beta(g)}{2 g} G_{\mu \nu}^{a} G^{a \mu \nu}\right\rangle_{T}=M_{0}(T) \\
\left\langle-\mathcal{S T} G_{\alpha \mu}^{a} G_{\beta}^{a \mu}\right\rangle_{T}=\left(u_{\alpha} u_{\beta}-\frac{1}{4} g_{\alpha \beta}\right) M_{2}(T) .
\end{gathered}
$$

Here, the symbol $\mathcal{S} \mathcal{T}$ denotes the traceless and symmetric part of the operator. We assign to $M_{0}(T)$ only the temperaturedependent part of the expectation value; it has, in general, a temperature-independent part, which is nothing but the gluon condensate in the vacuum.

The above equations immediately relate $M_{0}$ and $M_{2}$ to the thermodynamic quantities via the energy-momentum tensor in thermal equilibrium; namely, $M_{0}(T)=\varepsilon-3 p$ and $M_{2}(T)=$ $\varepsilon+p$ with $\varepsilon$ and $p$ being the energy density and the pressure respectively in the case of pure gluonic system [13]. In the presence of fermions, however, it is not straightforward to relate $M_{0}$ and $M_{2}$ to the thermodynamic quantities since the energy-momentum tensor has a fermionic part. The trace anomaly receives contributions from massive fermions as

$$
\left\langle T_{\mu}^{\mu}\right\rangle=\left\langle\frac{\beta(g)}{2 g} G_{\mu \nu}^{a} G^{a \mu \nu}\right\rangle+\sum_{i} m_{i}\left\langle\bar{q}_{i} q_{i}\right\rangle .
$$

$M_{0}$ becomes $\varepsilon-3 p$ only when the second term is negligible; otherwise, the fermionic part has to be explicitly subtracted out from $\varepsilon-3 p$ before identifying it to $M_{0}$. In lattice QCD calculations, contributions from each term in Eq. (3) have been estimated [20,21]. ${ }^{1}$ Similarly, $M_{2}$ can be related to the off-diagonal part of the energy-momentum tensor after the fermionic part is subtracted out. Such data are not yet available. Therefore, we need a scheme to subtract the fermionic contribution from the total of the energy-momentum tensor.

Going back to the original definition given in Eq. (2), one can relate the nucleon expectation values to $M_{0}$ and $M_{2}$ within the linear density approximation [26]

$$
\begin{gathered}
M_{0}^{\text {n.m. }}=\rho m_{N}^{0}, \\
M_{2}^{\text {n.m. }}=\rho A_{G} m_{N},
\end{gathered}
$$

\footnotetext{
${ }^{1}$ In Ref. [17], however, a somewhat different scheme is used to calculate the trace anomaly.
}

where $\rho, m_{N}^{0}, A_{G}$, and $m_{N}$ are the density of nucleus, the nucleon mass in the chiral limit, the second moment of gluon distribution function of the nucleon, and the nucleon mass, respectively. One sees in Eq. (4) that the chiral limit is taken for the nucleon mass, which corresponds to removing the fermionic term in the trace anomaly [Eq. (3)]. In Eq. (5), $A_{G}$ plays a similar role. Since these equations are expressed in terms of the mass of the particle consisting of the medium and its number density, one can extend them to genuine hadronic matter as

$$
\begin{gathered}
M_{0}^{\mathrm{had}}=\sum_{i=\text { hadrons }} \rho_{i} m_{i}^{0} \\
M_{2}^{\text {had }}=\sum_{i=\text { hadrons }} \rho_{i} m_{i} A_{G}^{i} .
\end{gathered}
$$

The quantities with subscript $i$ denote hadronic counterparts for the nucleon values appearing in Eqs. (4) and (5). As a simple model, we use a hadron resonance gas, including all hadrons for which the quantum numbers are known as given in the Particle Data Group [27]. The number density $\rho_{i}$ is now generalized to a hadron gas and is calculated as a function of $T$ and $\mu_{B}$ as

$$
\rho_{i}=\frac{d_{i}}{2 \pi^{2}} \int_{0}^{\infty} \frac{p^{2} d p}{\exp \left[\left(\sqrt{p^{2}+m_{i}^{2}}-\mu_{i}\right) / T\right] \pm 1},
$$

where the sign is + for fermions and - for bosons, and $d_{i}$ is the degree of freedom of the $i$ th hadron. We take into account the baryon number conservation and strangeness conservation and neglect isospin chemical potential for simplicity. The strangeness chemical potential $\mu_{s}$ is determined from the neutrality condition $\sum_{i} \rho_{i} S_{i}=0$ [28].

In Eq. (6), the masses of hadrons in the chiral limit $m_{0}^{i}$ are needed. Note that strange quark contribution from Eq. (3) and its off-diagonal counterpart also have to be subtracted. Thus, we will work within the flavor SU(3) symmetric limit. At present, we cannot know all of hadron masses in the $m_{u}=m_{d}=m_{s}=0$ limit, especially those of the highly excited states. Therefore, for the masses in the three-flavor chiral limit, we use different masses only for the Goldstone bosons, ground-state octet and decuplet baryons, and keep the masses of other hadrons the same as their vacuum values. Detailed lattice studies on hadron masses, as done in Ref. [29] with physical quark masses, will be helpful for more accurate treatment. Specifically, we first put $m_{\pi}^{0}=m_{K}^{0}=0$ and $m_{N}^{0}=750 \mathrm{MeV}$ from heavy baryon chiral perturbation theory [30]; these are the most important inputs needed for the masses in the chiral limit as the contributions to the thermodynamic quantities are dominated by these hadrons, especially by the Nambu-Goldstone bosons. For the vector and axial vector mesons, we assume $m_{\rho}^{0}=m_{\rho}$ and $m_{a_{1}}^{0}=m_{a_{1}}$. We also assume $m_{\Delta}^{0}=m_{\Delta}$. Furthermore, we also put $m_{f_{0}}^{0}=m_{\sigma}^{0}$, $m_{\phi}^{0}=m_{\omega}^{0}=m_{K^{*}}^{0}=m_{\rho}^{0}, m_{\Lambda}^{0}=m_{\Xi}^{0}=m_{\Sigma}^{0}=m_{N}^{0}$, and $m_{\Sigma^{*}}^{0}=$ $m_{\Xi^{*}}^{0}=m_{\Omega}^{0}=m_{\Delta}^{0}$, according to the flavor SU(3) symmetry.

The second moment of the gluon distribution function is set to $A_{G}^{i}\left(8 m_{c}^{2}\right)=0.9$ for all the hadrons. Generally, it can differ among hadrons, but it can be shown that $A_{G}^{\pi}$ differs little from this value at such a high energy scale, where the 


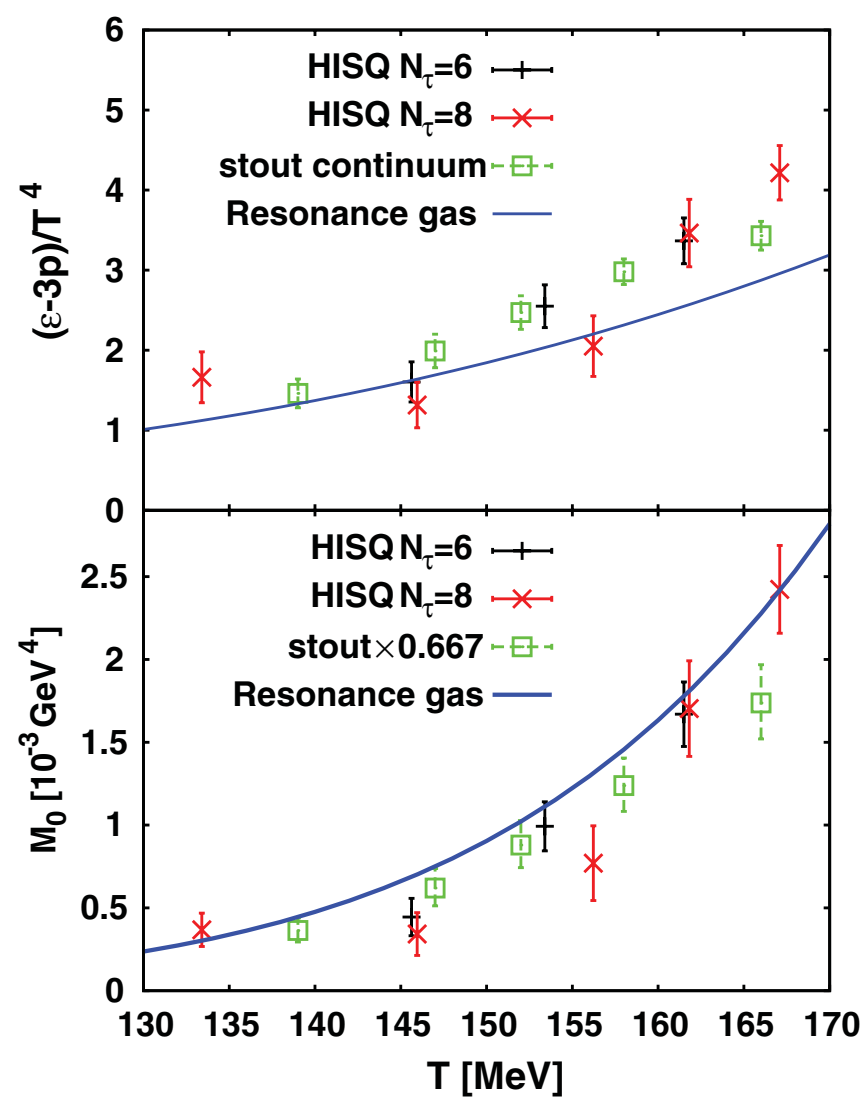

FIG. 1. (Color online) Comparison of the resonance gas with lattice results. Upper: interaction measure $(\varepsilon-3 p) / T^{4}$. Lower : its gluonic part $M_{0}$. Lattice data are taken from the HotQCD collaboration for HISQ action with $N_{\tau}=6$ and 8 [33] and from the "Budapest-Wuppertal" collaboration for "stout" action with continuum estimation (average of $N_{\tau}=8$ and 10) [17]. For $M_{0}$, stout data is estimated from $\varepsilon-3 p$ by assuming the same ratio of the gluonic part as that of HotQCD. See text for details.

parametrization of the gluon distribution functions $[31,32]$ are relatively well known.

The result of $M_{0}$ from the resonance gas model calculated with Eqs. (6) is shown in the lower panel of Fig. 1 together with $\varepsilon-3 p$ in the upper panel. We compare the resonance gas model with lattice data from two different fermion discretization schemes. One is from highly improved staggered fermion (HISQ) action, calculated by the HotQCD collaboration with temporal extent $N_{\tau}=6$ and 8 [33]. The other is from stout-link improved staggered fermion ("stout") action calculated by "Budapest-Wuppertal" collaboration [17]. In the former, the light quark mass is slightly heavier than the physical one, and the continuum extrapolation is not made, while the latter corresponds to physical quark masses and gives a continuum estimation. The upper panel shows the full trace anomaly, including both gluonic and fermionic parts for a reference. Lattice data obtained from the different schemes show reasonable agreement in the temperature range considered here. Therefore, we assume the present HISQ data already approximates the continuum result well. As already discussed in Ref. [17], the resonance gas model shows a small discrepancy at $T>150 \mathrm{MeV}$, which could be attributed to missing heavier states [34]. Since in our model $M_{0}$ is essentially dominated by light hadrons, we presume that this discrepancy does not affect the following analyses. In the lower panel, the HISQ data shows the gluonic part of the trace anomaly. Since the equation of state of the stout action was calculated in a different way, such that the gluonic part is not separated [17], we estimate it in the following way. First, we assume the ratio of the gluonic part of the trace anomaly to the total one is the same as those in the HISQ data. Next, we calculate the ratio by averaging that of HISQ data for $130 \mathrm{MeV}<T<170 \mathrm{MeV}$. The upper bound corresponds to upper limit of the pseudocritical temperature, below which we do not see clear temperature dependency in the ratio. Then we multiply $\varepsilon-3 p$ in the stout action by the resultant ratio factor 0.667 . Errors are estimated from the upper and lower value of the data points and from the average deviation of the ratio factor 0.052 of the HISQ data. One sees our model $M_{0}$ reproduces the lattice data well, and, therefore, we expect that the model gives a good approximation to $M_{2}{ }^{2}$ We display $M_{0}$ and $M_{2}$ as functions $T$ and $\mu_{B}$ in Fig. 2 .

In Fig. 2, we also draw the chemical freeze-out lines proposed by two groups. One (denoted by "FOI") is from a combined fit to statistical model results and has been shown to agree with various freeze-out conditions [23]. The temperature is given by

$$
T\left(\mu_{B}\right)=a-b \mu_{B}^{2}-c \mu_{B}^{4},
$$

where $a=0.166 \pm 0.002 \mathrm{GeV}, b=0.139 \pm 0.016 \mathrm{GeV}^{-1}$, and $c=0.053 \pm 0.021 \mathrm{GeV}^{-3}$. Collision-energy dependence is also given through the chemical potential

$$
\mu_{B}(\sqrt{s})=\frac{d}{1+e \sqrt{s_{N N}}},
$$

with $d=1.308 \pm 0.028 \mathrm{GeV}$ and $e=0.273 \pm 0.016 \mathrm{GeV}^{-1}$. It has been shown that this parametrization works well for recent STAR data [36].

The other (denoted by FOII) is a parametrization of results of a statistical model shown in Ref. [24]. The freeze-out temperature and chemical potential are given as functions of $\sqrt{s_{N N}}$ (in unit of $\mathrm{GeV}$ ):

$$
\begin{gathered}
T(\sqrt{s})=T_{\lim }\left(1-\frac{1}{0.7+\left(e^{\sqrt{s_{N N}}}-2.9\right) / 1.5}\right) \\
\mu_{B}(\sqrt{s})=\frac{a^{\prime}}{1+b^{\prime} \sqrt{s_{N N}}}
\end{gathered}
$$

where $T_{\lim }=161 \pm 4 \mathrm{MeV}, a^{\prime}=1303 \pm 120 \mathrm{MeV}$, and $b^{\prime}=$ $0.286 \pm 0.049 \mathrm{GeV}^{-1}$. We draw two lines for each freeze-out curve, corresponding to upper and lower temperatures estimated by uncertainties in the parameters. The main difference between the two freeze-out curves is seen at $\mu_{B}>300 \mathrm{MeV}$, which corresponds to $\sqrt{s_{N N}}<10 \mathrm{GeV}$. While the one by

\footnotetext{
${ }^{2}$ One may try to improve the agreement by introducing interactions in the resonance gas. For example, we can use an excluded volume correction to the resonance gas, which incorporates the repulsive interaction among hadrons [35]. We found, however, that $\chi^{2}$ fitting of the excluded volume parameter $v_{0}$ to the lattice data of $M_{0}$ and $\varepsilon-3 p$ gives a consistent result with $v_{0}=0$.
} 
(a)

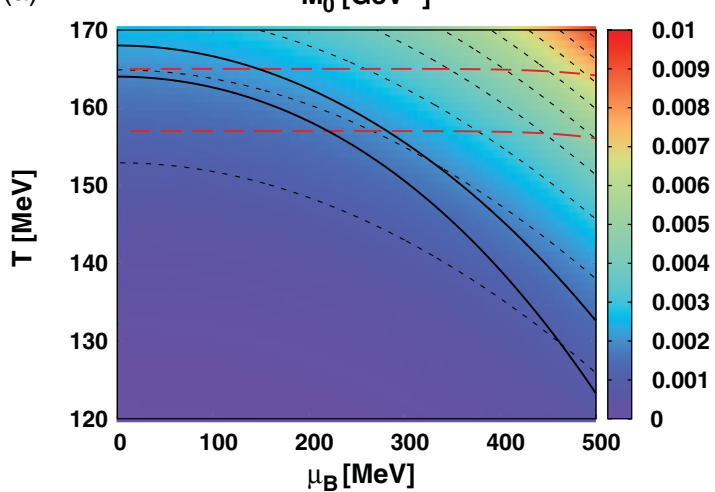

(b)

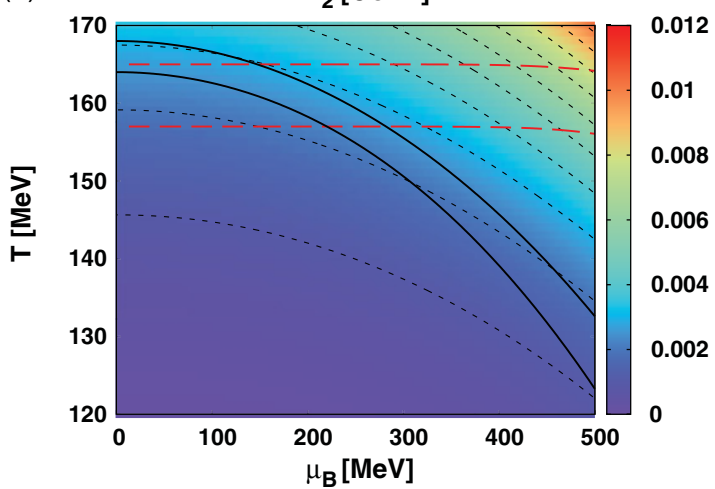

FIG. 2. (Color online) Contour plots for $M_{0}[(\mathrm{a})]$ and $M_{2}[(\mathrm{~b})]$ obtained from the resonance gas model, Eqs. (6) and (7). The thick solid lines (black) indicate the chemical freeze-out line, including uncertainty of parameters given in Ref. [23]. The thick dashed lines (red) also indicate the freeze-out line from Ref. [24].

Cleymans et al., FOI, has a strong curvature, which leads to lower freeze-out temperature in this region, and the other, by Andronic et al., FOII, shows almost constant freeze-out temperature up to $\mu_{B} \sim 500 \mathrm{MeV}$, resulting in coincidence with the QCD chiral transition line [37]. ${ }^{3}$ Although the difference between them seems to partly come from the fact that FOI uses $4 \pi$ particle yield while FOII uses midrapidity data only, one may consider the constant temperature case (FOII) to be simultaneous chemical freeze-out at the hadronization.

For charmonium production, it is not clear which scenario is more likely, due to small production rate at lower energies. At the top SPS energy, where the charmonium particle ratio data is available, $\sqrt{s_{N N}}=17.3 \mathrm{GeV}$, the two freeze-out curves coincide. From Fig. 2, one sees both $M_{0}$ and $M_{2}$ increases as $\mu_{B}$ does so at fixed temperature. This implies larger medium modification of charmonia for larger chemical potential. If the freeze-out temperature decreases steeper, as in FOI, however, resultant $M_{0}$ and $M_{2}$ do not differ so much. Therefore, we expect that the mass shift of charmonium substantially differs at lower collision energies between the two possible freeze-out scenarios.

\footnotetext{
${ }^{3}$ Although this line corresponds to the chiral transition, it can presumably represent the deconfinement transition line also.
}

\section{MASS SHIFT OF CHARMONIUM}

\section{A. Second-order Stark effect}

First, we calculate the mass shift of $J / \psi$ using the secondorder Stark effect in QCD as done in Ref. [13]. Provided the wave function of the quarkonium in the momentum space $\psi(k)$ is normalized as $\int \frac{d^{3} \boldsymbol{k}}{(2 \pi)^{3}}|\psi(\boldsymbol{k})|^{2}=1$, the formula of the mass shift for the $1 S$ state is given by $[13,38-40]$

$$
\begin{aligned}
\Delta m_{J / \psi} & =-\frac{1}{18} \int_{0}^{\infty} \frac{k d k^{2}}{k^{2} / m_{c}+\epsilon}\left|\frac{\partial \psi(k)}{\partial k}\right|^{2}\left\langle\frac{\alpha_{s}}{\pi} \Delta \boldsymbol{E}^{2}\right\rangle_{T, \mu_{B}} \\
& =-\frac{7 \pi^{2}}{18} \frac{a^{2}}{\epsilon}\left\langle\frac{\alpha_{s}}{\pi} \Delta \boldsymbol{E}^{2}\right\rangle_{T, \mu_{B}}
\end{aligned}
$$

where $k=|\boldsymbol{k}|$ and $m_{c}$ and $\epsilon$ are the charm quark mass and the binding energy, respectively. The above formula can be also derived from potential nonrelativistic QCD (pNRQCD), as shown in the Appendix. The second line is obtained for the Coulombic bound state with Bohr radius $a$. These parameters can be determined by a fit to the $J / \psi$ mass in vacuum and the size of the wave function in the Cornell potential model [3]. It gives $m_{c}=1704 \mathrm{MeV}, a=0.271 \mathrm{fm}$, and $\alpha_{s}=0.57$. In this formula, the mass shift is proportional to the change of the electric condensate $\left\langle\frac{\alpha_{s}}{\pi} \Delta \boldsymbol{E}^{2}\right\rangle_{T, \mu_{B}}$ from its vacuum value. The electric condensate as well as the magnetic counterpart can be written in terms of $M_{0}$ and $M_{2}$ as [13], for $N_{f}=3$,

$$
\begin{gathered}
\left\langle\frac{\alpha_{s}}{\pi} \Delta \boldsymbol{E}^{2}\right\rangle_{T, \mu_{B}}=\frac{2}{9} M_{0}\left(T, \mu_{B}\right)+\frac{3}{4} \frac{\alpha_{s}^{\mathrm{eff}}}{\pi} M_{2}\left(T, \mu_{B}\right), \\
\left\langle\frac{\alpha_{s}}{\pi} \Delta \boldsymbol{B}^{2}\right\rangle_{T, \mu_{B}}=-\frac{2}{9} M_{0}\left(T, \mu_{B}\right)+\frac{3}{4} \frac{\alpha_{s}^{\mathrm{eff}}}{\pi} M_{2}\left(T, \mu_{B}\right) .
\end{gathered}
$$

Here, the effective coupling constant $\alpha_{s}^{\text {eff }}$ can be chosen according to the relevant energy scale to the expectation value of the operator. In this case, the formula is based on OPE with separation scale $\epsilon$. Thus, it is plausible to take $\alpha_{s}^{\text {eff }}=0.57$ obtained from the fit to the bound state.

Figure 3 shows the electric condensate for $\mu_{B}=$ $0,100,200,300$, and $400 \mathrm{MeV}$ as a function of temperature. The maximum chemical potential $400 \mathrm{MeV}$ roughly corresponds to $40 A \mathrm{GeV} \mathrm{Pb}+\mathrm{Pb}$ collisions at SPS, the lowest collision energy above the $J / \psi$ production threshold [41]. One sees a larger change of the electric condensate for high temperature and chemical potential as is expected from Fig. 2. Now we are able to estimate the mass shift of $J / \psi$ by putting the condensate into Eq. (13). We compute the mass shift along the freeze-out lines shown in Fig. 2 and show the result as a function of $\sqrt{s_{N N}}$ in Fig. 4. For higher colliding energies than $\sqrt{s_{N N}}>30 \mathrm{GeV}$, the mass shift is independent of the colliding energy owing to the fact that the freeze-out temperature varies little and the chemical potential does not change the condensate significantly (see Fig. 3). The amount of the downward mass shift is $10-20 \mathrm{MeV}$, including uncertainty. On the other hand, low-energy results differ between FOI and FOII, as expected from Fig. 2. Along the FOI chemical freeze-out line, the mass shift becomes smaller while opposite behavior is seen for FOII. In the FOII case, since temperature is almost constant, the larger the chemical potential, the bigger the mass shift 


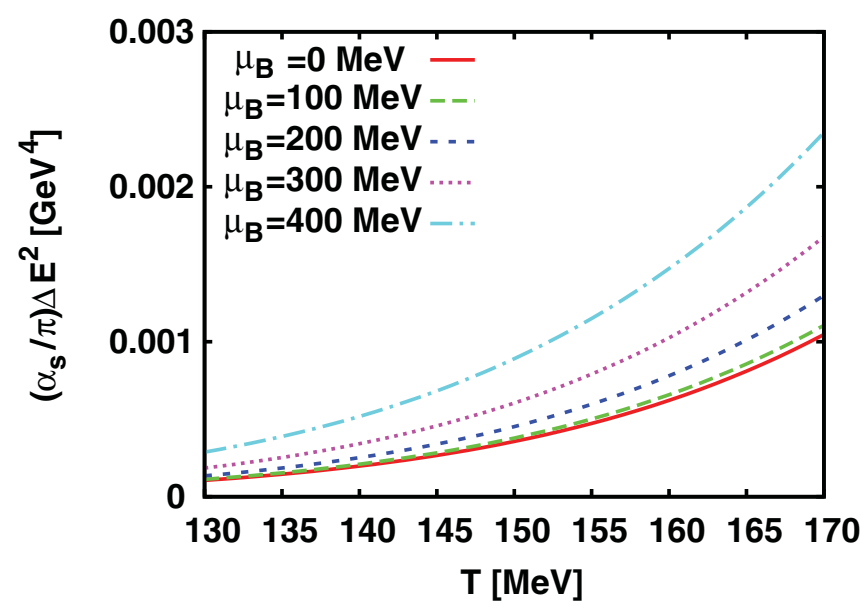

FIG. 3. (Color online) Temperature-dependent part of the electric condensate $\left\langle\frac{\alpha_{s}}{\pi} \Delta \boldsymbol{E}^{2}\right\rangle$. Each line stands for the case of different chemical potential.

becomes, owing to the effects from the chemical potential. In the other case, however, decreasing freeze-out temperature cancels the effect of the chemical potential. For instance, one can see in Fig. 3 that $\Delta \boldsymbol{E}^{2}$ at $T=165 \mathrm{MeV}$ and $\mu_{B}=0$ is almost equal to that at $T=145 \mathrm{MeV}$ and $\mu_{B}=400 \mathrm{MeV}$. Therefore, At the lowest SPS energy, the downward mass shift ranges from 10 to $60 \mathrm{MeV}$, depending on the choice of the thermal parameters.

\section{B. QCD sum rules}

While the second-order Stark effect provides the downward mass shift directly in the case of the increasing electric condensate, QCD sum rule is expected to be more quantitatively reliable according to the larger separation scale by going to the deep Euclidean region. Here we give an estimation based on

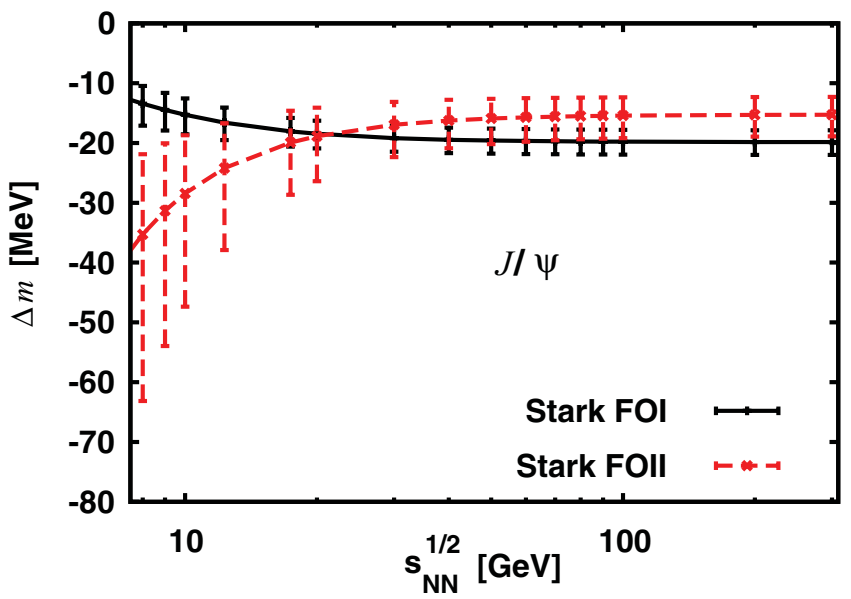

FIG. 4. (Color online) Mass shift of $J / \psi$ at freeze-out temperature and chemical potential from the second-order Stark effect. The horizontal axis denotes the collision energy, which is related to the temperature and the chemical potential via Eqs. (9) and (10) for the FOI case and Eqs. (11) and (12) for the FOII case. the Borel sum rule framework used in Ref. [14] with further improvement as described below.

The two-point current correlation function in the vector channel after the Borel transformation with the Borel mass $M^{2}$ is given by $[14,42,43]$

$$
\begin{aligned}
\mathcal{M}\left(M^{2}\right)= & e^{-v} \pi A(v)\left[1+\alpha_{s}\left(M^{2}\right) a(v)\right. \\
& \left.+b(v) \phi_{b}(T)+c(v) \phi_{c}(T)\right],
\end{aligned}
$$

with $v=4 m_{c}^{2} / M^{2}$. Here, the system is assumed to be at rest with respect to the medium. The Wilson coefficients $a(v), b(v)$, and $c(v)$ are listed in Ref. [14]. The temperature dependency is governed by the dimension four gluon condensate terms $\phi_{b}(T)$ and $\phi_{c}(T)$ given by

$$
\begin{aligned}
\phi_{b} & =\frac{4 \pi^{2}}{9\left(4 m_{c}^{2}\right)^{2}} G_{0}(T), \\
\phi_{c} & =\frac{4 \pi^{2}}{3\left(4 m_{c}^{2}\right)^{2}} G_{2}(T) .
\end{aligned}
$$

The relations of the positive definite quantities $M_{0}$ and $M_{2}$ to the dimension four gluon condensates appearing in the OPE side correlation function are given by

$$
\begin{aligned}
& G_{0}(T)=G_{0}^{\mathrm{vac}}-\frac{8}{9} M_{0}(T) \\
& G_{2}(T)=-\frac{\alpha_{s}^{\mathrm{eff}}}{\pi} M_{2}(T)
\end{aligned}
$$

after taking the one-loop expression for the beta function. As in the pure gauge case, we use a temperature-dependent effective coupling constant $\alpha_{s}^{\text {eff }}=\alpha_{q q}(T)$, extracted from the lattice calculation of the color singlet heavy quark free energy by assigning $\alpha_{s}\left\langle G_{\mu \rho}^{a} G^{a v \rho}\right\rangle_{T} \equiv\left\langle\alpha_{s}(T) G_{\mu \rho}^{a} G^{a v \rho}\right\rangle$ in the spirit of the separation scale in the heavy quark system, which imposes all the temperature effect on the condensates [13,22]. We take the values from $N_{f}=2$ results of $\alpha_{q q}\left(r_{\max }\right)$ in Fig. 6 of Ref. [44]. To account for different critical temperatures between the $N_{f}=2$ simulation, $T_{c}=202 \mathrm{MeV}$, and the reality (see Sec. I), we rescale the coupling constant from the data by assuming similar temperature dependency to that of $T_{c}=170 \mathrm{MeV}$. This value is considered to be the upper bound of the pseudocritical temperature in reality, owing to a measurement based on the strange quark number susceptibility $T_{p c}=165(5)(3) \mathrm{MeV}$ in Ref. [19]

Presumably, this choice does not affect the results quantitatively, since the twist- 2 contribution to the medium modification is relatively small in the hadronic phase [11]. The $T=0$ part of the scalar gluon condensate $G_{0}^{\mathrm{vac}}$ is fixed to be $(0.35 \mathrm{GeV})^{4}[45]$ as in our previous calculations. ${ }^{4}$

The Borel-transformed correlation function is related to the spectral density through the dispersion relation

$$
\mathcal{M}\left(M^{2}\right)=\int_{0}^{\infty} d s e^{-s / M^{2}} \operatorname{Im} \tilde{\Pi}(s) .
$$

\footnotetext{
${ }^{4} G_{0}^{\mathrm{vac}}$ has still a large error after fitting to the various experimental data; see also Ref. [46] for example. This vacuum value, however, does not affect the in-medium effect significantly, since we are looking at relative changes from vacuum.
} 
We model the right-hand side of the dispersion relation with a simple ansatz and call it the phenomenological side as usual. In the hadronic medium below $T_{c}$, previous analyses based on the gluon condensate of pure gauge theory indicates the broadening is small enough to ignore. Provided the continuum part of the model spectral density $\mathcal{M}^{\text {cont }}\left(M^{2}\right)$, the the mass of $J / \psi$ is given by

$$
m_{J / \psi}^{2}\left(M^{2}\right)=-\frac{\frac{\partial}{\partial\left(1 / M^{2}\right)}\left[\mathcal{M}\left(M^{2}\right)-\mathcal{M}^{\text {cont }}\left(M^{2}\right)\right]}{\mathcal{M}\left(M^{2}\right)-\mathcal{M}^{\operatorname{cont}}\left(M^{2}\right)} .
$$

We use the perturbative expression up to $\mathcal{O}\left(\alpha_{s}\right)$ for $\mathcal{M}^{\text {cont }}\left(M^{2}\right)$ as in Ref. [14].

Since the mass of $J / \psi$ is a function of the Borel mass $M^{2}$, which is an unphysical parameter, one has to choose the range of $M^{2}$ called Borel window by following the criteria:

(i) $M_{\min }^{2}$ : Convergence of the OPE by imposing the dimension four operator contribution less than $30 \%$ to the total OPE [47];

(ii) $M_{\max }^{2}$ : Continuum contribution to the dispersion integral is less than $30 \%$. We choose the threshold parameter $s_{0}$ such that extracted $J / \psi$ mass is least sensitive to $M^{2}$.

As discussed in Ref. [14], the values $30 \%$ are physically reasonable but arbitrary. Due to truncation of the OPE, we cannot obtain the completely $M^{2}$-independent mass. Specifically, the mass strongly varies with $M^{2}$ at lower $M^{2}$ even inside the Borel window. As this can be regarded as a systematic uncertainty due to the truncation, we take this effect into account in the mass evaluation by averaging the mass over the Borel window and take its variance as the error [22]. Namely,

$$
\bar{m}=\int_{M_{\min }^{2 \prime}}^{M_{\max }^{2}} d M^{2} m\left(M^{2}\right) /\left(M_{\max }^{2}-M_{\min }^{2 \prime}\right)
$$

and

$$
(\delta m)^{2}=\int_{M_{\min }^{2 \prime}}^{M_{\max }^{2}} d M^{2}\left(m\left(M^{2}\right)-\bar{m}\right)^{2} /\left(M_{\max }^{2}-M_{\min }^{2 \prime}\right) .
$$

An example taken from $T=0$ is shown in Fig. 5. Here, the Borel window is defined by $M^{2} \in\left[M_{\min }^{2 \prime}, M_{\max }^{2}\right]$ and $M_{\min }^{2 \prime} \equiv$ $\max \left(M_{\min }^{2}, M_{0}^{2}\right)$.

We have introduced $M_{0}^{2}$ such that $\operatorname{dm}\left(M^{2} ; \sqrt{s_{0}}=\right.$ $\infty) / d M^{2}=0$ in order to remove the strongly $M^{2}$-dependent part of $m\left(M^{2}\right)$ from the evaluation of the average [Eq. (22)] and variance [Eq. (23)]. This is a reasonable choice as the continuum threshold is so determined that it makes the Borel curve flattest at $M^{2}>M_{0}^{2}$.

Figure 5 shows an example of the determination process. We start with $\sqrt{s_{0}}=\infty$ case, which gives $M_{0}^{2}$ shown as the dashed line. Then we search for $\sqrt{s_{0}}$ such that it gives the smallest $\delta m$ using Eq. (23), which takes its minimum when the deviation from the average value is the smallest. The resultant average deviation is indicated by the band in the figure. Irrespective to the temperature, it is found to be approximately 5 and $6 \mathrm{MeV}$ in the case of $J / \psi$ and $\chi_{c 1}$, respectively. Parameters of the theory are fixed to $m_{c}\left(p^{2}=\right.$

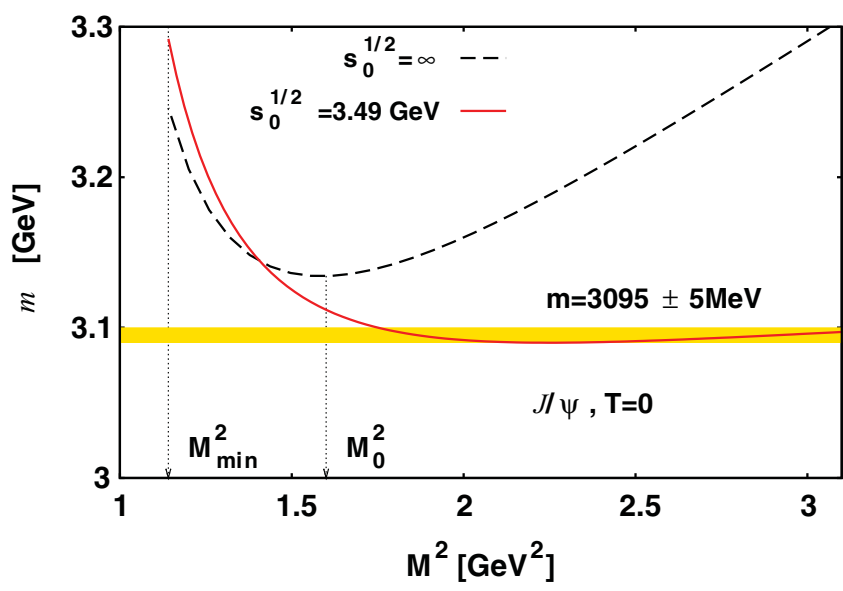

FIG. 5. (Color online) Borel curves at $T=0$. The dashed line stands for the case of $\sqrt{s_{0}}=\infty$. The solid line denotes the case of $\sqrt{s_{0}}=3.49 \mathrm{GeV}$, which gives the flattest curve according to Eq. (23). The band indicates the systematic uncertainty associated with the flattest Borel curve.

$\left.-2 m_{c}^{2}\right)=1.262 \mathrm{GeV}$ and $\alpha_{s}\left(8 m_{c}^{2}\right)=0.21$ by fitting to the vacuum $J / \psi$ and $\chi_{c 1}$ masses on the basis of the same criterion of the Borel window. This process removes the ambiguity on the arbitrary choice of the criterion mentioned above. Including the width is straightforward. As shown in Ref. [14], introducing width increases the mass at small $M^{2}$. This fact leads to larger $s_{0}$ after minimizing $\delta m$. Then we will have the mass-width relation similar to those shown in Refs. [10,11,14]. In most cases, $M_{0}^{2}>M_{\mathrm{min}}^{2}$ holds in the charmonium sum rules. At high temperature and chemical potential, however, we found that the Borel stability is lost [10,14]; thus, $M_{0}^{2}$ is not well defined. In such cases, we can still recover the Borel stability by decreasing the threshold parameter or by introducing the width. When the width must be introduced, we cannot determine both mass and width simultaneously but have only constraints. In what follows, we restrict ourselves to cases in which the Borel stability is established with vanishing width.

Figure 6 displays the results of the mass shift obtained from the QCD sum rule analysis as described above. We plot the mass shifts corresponding to the two freeze-out curves as in the Stark effect results (Fig. 4). Errors are estimated from the uncertainty in the thermal parameters in Eqs. (9)(12). The systematic errors in the Borel sum rules, which have been introduced above, are not included in the plot. We also calculate mass shift of $\chi_{c 1}$ in the same way. In $\chi_{c}$, we have observed the loss of the Borel stability at lower collision energies than $\sqrt{s_{N N}}<8 \mathrm{GeV}$ in FOII, owing to much change of the gluon condensate [14]. Comparing the result with Fig. 4, one finds that the two methods give consistent mass shifts as found in Ref. [14]. The mass shift of $\chi_{c 1}$ is approximately twice as large as that of $J / \psi$, as previously found $[12,14]$. We expect similar results for other $\chi_{c}$ states.

Before closing the section, we would like to comment on the effect of the scattering term, which was pointed out in Ref. [48] to appear as a pole at the zero mode in the correlator. In our previous works, it was neglected since 


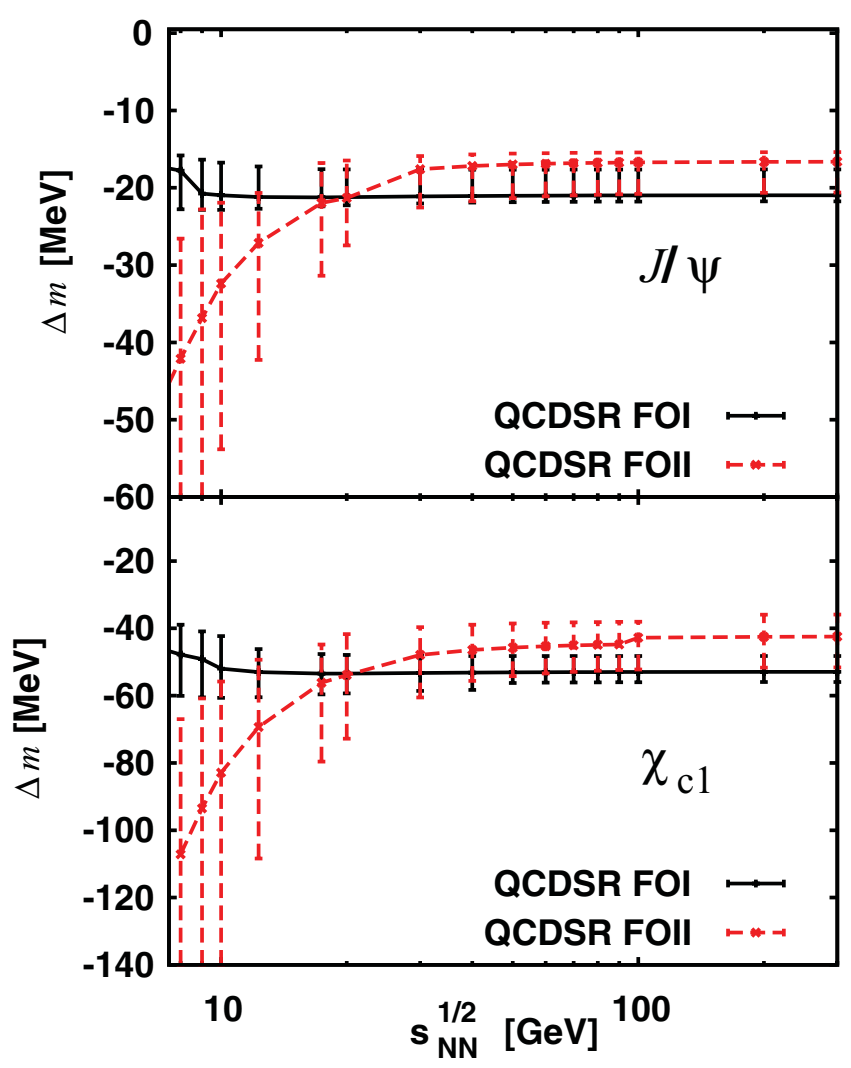

FIG. 6. (Color online) Mass shift of $J / \psi$ (upper) and $\chi_{c 1}$ (lower) obtained with QCD sum rules. Solid and dashed lines stand for the mass shift corresponding to the different freeze-out curves, as in Fig. 4. Errors are calculated from the uncertainty in the thermal parameters.

such a contribution appears in the OPE side to cancel the phenomenological side put as an ansatz. In the deconfined phase, this argument should hold because the physical particle absorbing the current is the (anti)charm quark, while this is not so in the hadronic phase, where charmed mesons are the physical particles. In general, these terms can be neglected as they contribute at zero energy in the spectral density. However, without invoking such arguments, it can be neglected in the present case on the following grounds. First, the scattering terms in the OPE and the phenomenological side will be proportional to $e^{-m_{c} / T}$ and $e^{-m_{D} / T}$, respectively, while the other OPE terms in the Borel transformed sum rule will in general scale as $e^{-4 m_{c}^{2} / M^{2}}$. Hence, as long as $T<M^{2} /\left(4 m_{c}\right)$ or $T<M^{2} m_{D} /\left(4 m_{c}^{2}\right)$, the scattering terms can be neglected. Since the smallest Borel mass relevant in our analysis is always larger than $1 \mathrm{GeV}$, taking $m_{c}=1.26 \mathrm{GeV}$, one finds that the scattering terms can be safely neglected for $T<200 \mathrm{MeV}$. Moreover, the open charm meson will receive greater medium effect than charmonia, making $m_{D}$ close to $m_{c}$. Thus, the scattering contribution from the OPE and the phenomenological side will tend to cancel each other. Second, one can remove the scattering contribution by making use of the fact that it contributes as a constant term to the Borel transformed correlator $\mathcal{M}\left(M^{2}\right)=\int d s e^{-s / M^{2}} \rho(s)$, since the scattering contribution to the spectral density takes

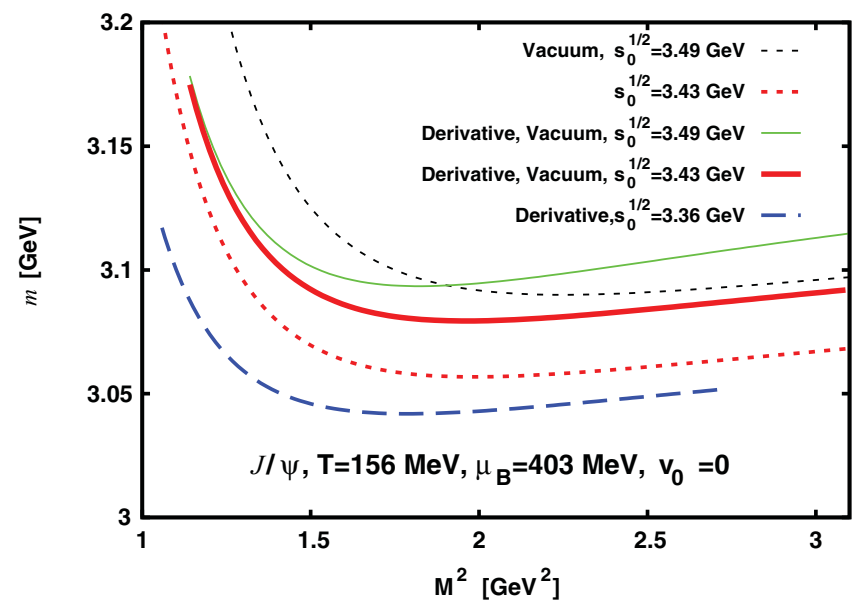

FIG. 7. (Color online) Borel curves for the mass obtained from the ordinary QCD sum rule and from the differentiated one, Eq. (24). See text for detailed explanation.

a form as $\rho^{\text {scat }}(s) \propto \delta(s)$ [48](equivalently $\sqrt{s} \delta(\sqrt{s})$ [49-51]). Therefore, the effect of the scattering term does not exist in the derivative of $\mathcal{M}\left(M^{2}\right)$ with respect to $M^{2}\left(1 / M^{2}\right.$ in practical calculations). One may then start from the once differentiated sum rule and express the mass in the $\Gamma=0$ limit as

$$
m_{c \bar{c}}^{2}=\frac{\frac{\partial^{2}}{\partial\left(1 / M^{2}\right)^{2}}\left[\mathcal{M}\left(M^{2}\right)-\mathcal{M}^{\mathrm{cont}}\left(M^{2}\right)\right]}{-\frac{\partial}{\partial\left(1 / M^{2}\right)}\left[\mathcal{M}\left(M^{2}\right)-\mathcal{M}^{\mathrm{cont}}\left(M^{2}\right)\right]} .
$$

Indeed, such a method was advocated in Ref. [52] but criticized in Ref. [53] because starting with a higher-order derivative makes the OPE side more sensitive to unknown higher-dimensional condensates and Borel stability is lost; it was claimed in Ref. [53] that due to this artifact, the light vector meson was found to increase in the medium in Ref. [52]. This comes from the fact that the OPE in the light vector meson has no scale parameter other than the Borel mass; therefore, after the Borel transformation, the sum rule becomes a polynomial in $1 / M^{2}$ with the highest power determined by the highest dimensional operator calculated in the OPE. In the case of heavy quarkonia, however, the presence of heavy quark mass does not make the OPE a mere polynomial in $1 / M^{2}$, so that stability is not lost even after derivatives.

Figure 7 shows evidence for the above argument. We show the Borel curves obtained from Eq. (24) as well as those from the ordinary method, Eq. (21). For illustration, we show a case with large medium effect, corresponding to $\sqrt{s_{N N}}=8.7 \mathrm{GeV}$ in FOII, of which temperature and chemical potential are $T=$ $156 \mathrm{MeV}$ and $\mu_{B}=403 \mathrm{MeV}$, respectively. For vacuum, we display three curves. The thin dotted (black) curve shows the same one as in Fig. 5 for the reference. The thin solid (green) curve denotes that obtained from Eq. (24) with the same value of $\sqrt{s_{0}}$. One sees both curves give almost the same mass. After minimizing $\delta m$ in the differentiated sum rule [Eq. (24)], one gets a slightly smaller mass indicated by the thick solid (red) line. One notes Eq. (24) gives smaller mass at small $M^{2}$, as expected from the fact that it becomes sensitive to higher-dimensional operators. The small discrepancy of the mass can be attributed to the dimension six contribution, which 
slightly increases the mass [54]. The remaining two curves are for the medium. The thick dotted (red) curve is obtained from the ordinary sum rule while the thick dashed (blue) curve is from the derivative with the optimization. If one compares them with the corresponding results for vacuum, one finds that the mass shifts are almost the same between the two sum rules. Hence, we conclude the general properties of the in-medium modification of heavy quarkonia at low temperature up to near $T \simeq 160 \mathrm{MeV}$ will not change by including the scattering contribution.

\section{IMPLICATION FOR EXPERIMENTS}

We have discussed mass shifts of $J / \psi$ in hot medium, which could be produced in heavy ion collisions. Production mechanism of $J / \psi$ has not been fully understood yet because of the still unknown elementary production process and also the complicated collision processes [55]. In the following, we briefly review the collision process and specify the situation we will consider in this section. Charmonia have been considered to be mostly produced by collisions between initial state quarks and gluons in nuclei at the initial stage of heavy ion collisions. The formation time scale $\tau \sim 1 /\left(2 m_{c}\right)$ is supposed to be shorter than the thermalization time scale of the medium, which is related to flow measurements through hydrodynamic model calculations. The produced charmonia will also interact with colliding nuclei. The dissociation of $J / \psi$ by this interaction, called cold nuclear matter effect, is estimated by the nuclear absorption cross section, which is roughly $1.5 \mathrm{mb}$ at RHIC energies and $4.4 \mathrm{mb}$ at SPS energies [56]. In the hot medium, charmonia could melt. While Lattice QCD have shown existence of the spectral peak even at higher temperature $[4,5,7,8]$, model calculations can explain the lattice data with melting of $J / \psi$ [57]. Even if the bound states can survive, they will acquire substantial collisional broadening through interacting with quarks and gluons in medium $[55,58]$. There could be also recombination of a $c \bar{c}$ pair inside deconfined medium below dissociation temperature. Finally, (anti-)charm quarks hadronize at the phase boundary to form charmed mesons, baryons, and hidden charm states. If $J / \psi$ mass is modified in the medium and decay inside the mediun, one may be able to observe it as modification of the peak in dilepton channel. However, one needs a dynamical approach in order to take into account various processes described above, as well as microscopic information like cross sections to estimate the final yield $[2,55,59]$.

Here, we consider an alternative possibility of indirect observation via statistical production [25,41,60-62]. This has been already considered as a part of the contribution in the transport approach and could give substantial contribution to the final yields at high centrality [55]. In Ref. [41], in-medium effect on the charmonium yields was considered as a result of $D$ meson mass modification and charm conservation. As emphasized in the literature, charm conservation plays an important role in the statistical description of charm quarks. However, as for the particle ratio between charmonia, the fugacity factor cancels and the ratio can be expressed as that of thermal number densities at given temperature and chemical potential. Therefore, we can focus on observables dominated by the statistical production.

First we examine $N_{\psi^{\prime}} / N_{J / \psi}$ at midrapidity, which was also investigated in Ref. [25], since there was experimental data for $\mathrm{Pb}+\mathrm{Pb}$ collisions at $\sqrt{s_{N N}}=17.3 \mathrm{GeV}$ in CERN-SPS. The temperature and chemical potential in the two freeze-out curves coincide at this energy as seen from Fig. 2. We adopt $T=160 \mathrm{MeV}$ and $\mu_{B}=240 \mathrm{MeV}$ in the following [25] and discuss possible effects of charmonium mass shifts.

Although the QCD sum rule method cannot assess inmedium modification of $\psi^{\prime}, 5$ it is expected to be strongly affected [63]. While applicability of the formula of the secondorder Stark effect in Ref. [38] is questionable for the physical $\psi^{\prime}$, a crude estimation based on the dipole nature might be possible. If we assume the mass shift scales with the size of the wave function, the mass shift of $\psi^{\prime}$ is a factor 4.2 larger than that of $J / \psi$. For the SPS data at $\sqrt{s_{N N}}=17.3 \mathrm{GeV}$, which we will analyze below, $\Delta m_{\psi^{\prime}}$ becomes $63-119 \mathrm{MeV}$ by adopting the result from QCD sum rules and fully taking the errors into account. Here, we vary $\psi^{\prime}$ mass shift in a broader range than the above estimation and calculate the ratio as a function of $\Delta m_{\psi^{\prime}}$. The total number of $J / \psi$ is obtained by summing up decay contributions from $\psi^{\prime}$ and $\chi_{c J}(J=0,1,2)$. We assume the mass shifts of different $\chi_{c}$ states to be the same as that of $\chi_{c 1}$ and the branching ratios to be the same as their vacuum values. Specifically, the branching ratios of $\psi^{\prime}$ and $\chi_{c J}(J=0,1,2)$ to $J / \psi$ are taken to be 0.595 , $0.0116,0.344$, and 0.195 , respectively [27].

We plot the result for $T=160 \mathrm{MeV}$ and $\mu_{B}=240 \mathrm{MeV}$ together with experimental data taken from $\mathrm{Pb}+\mathrm{Pb}$ collisions at $\sqrt{s_{N N}}=17.3 \mathrm{GeV}$ [64] in the lower panel of Fig. 8 . One sees that a strong variation of the ratio as a function of $\psi^{\prime}$ mass shift. The band for our result corresponds to the uncertainty in the mass shift shown in Fig. 6. If the $\psi^{\prime}$ downward mass shift is smaller than $100 \mathrm{MeV}$, which is a reasonable value according to the crude estimation, the expected mass shifts are consistent with the experimental data. At present, however, it seems difficult to draw a conclusion from this observation; in-medium modification of $\psi^{\prime}$ is still controversial. While the ground state, $J / \psi$, seems to exist in the vicinity of the transition, $\psi^{\prime}$ can be dissociated at finite temperature even below transition temperature according to potential model calculations [9]. Even if $\psi^{\prime}$ is statistically produced with the large mass reduction, it can still be dissolved into charmed mesons in hadronic medium thus observed yields might be smaller than what was produced at the hadronization: the dissociation cross section of $J / \psi$ in hadronic matter is expected to be smaller than that of $\psi^{\prime}$ due to its smaller size [59]. In this case, there must be, of course, enhancement of charmed mesons due to charm conservation. The analysis might become complicated if initially produced

\footnotetext{
${ }^{5}$ In the Borel transformed sum rule, we can obtain the same $J / \psi$ mass even if we incorporate the $\psi^{\prime}$ contribution in the model spectral function explicitly [14]. Therefore, in-medium effect on $\psi^{\prime}$ is incorporated in the effective threshold parameter $\sqrt{s_{0}}$. This shows the strong sensitivity of the sum rule to the lowest pole of the spectral function.
} 


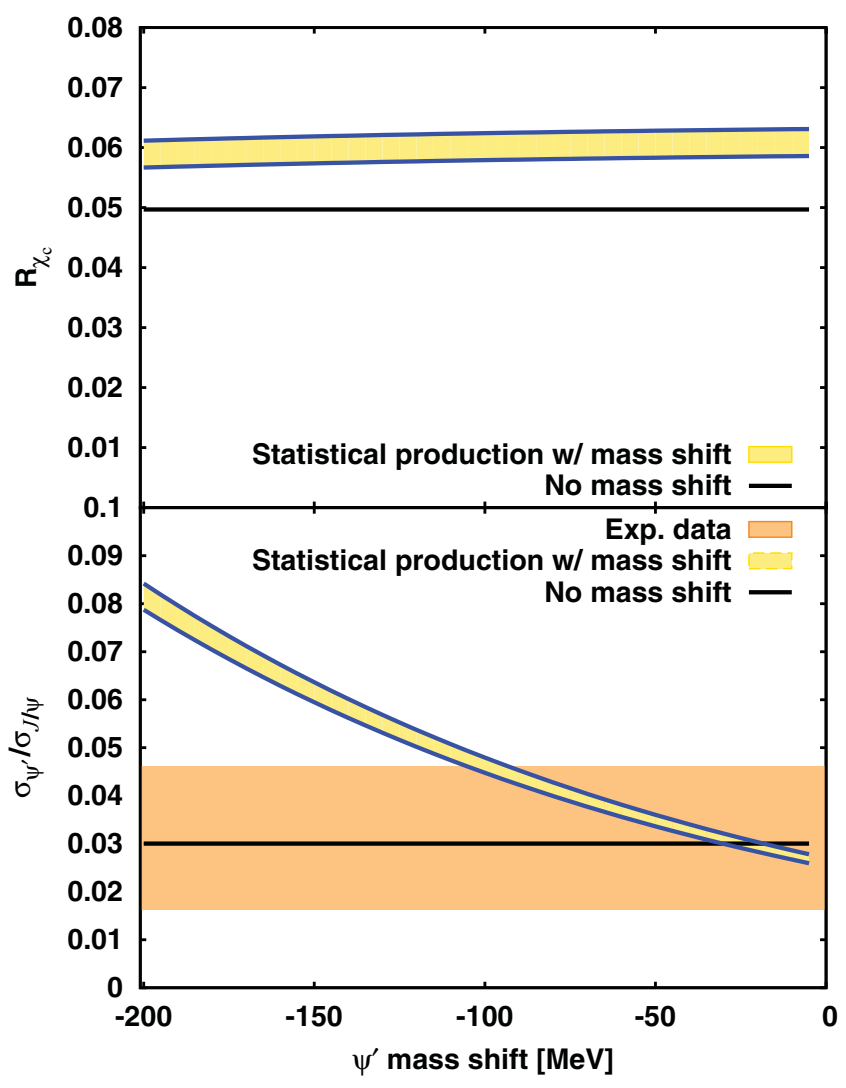

FIG. 8. (Color online) Particle number ratio at $T=160 \mathrm{MeV}$ and $\mu_{B}=240 \mathrm{MeV}$ as a function of $\psi^{\prime}$ mass shift. The band in the lower panel indicates the experimental data taken from $\mathrm{Pb}+\mathrm{Pb}$ collisions at $\sqrt{s_{N N}}=17.3 \mathrm{GeV}$ measured by NA50 collaboration [64]. The solid lines stand for the results without any mass shift in the charmonia for comparison.

charmonium could survive the quark-gluon plasma phase and the hadron phase. Then there will be modification to statistical model prediction as discussed in a two-component model of charmonium production in a heavy ion collision $[59,65]$. However, even within the two-component model, the thermal production will be dominant at LHC and production ratios can reveal vital information at the hadronization point.

Although experimentally challenging, a more promising observable could be the ratio of $\chi_{c}$ to $J / \psi$. In the upper panel, we display a fraction of $J / \psi$ coming from $\chi_{c 1}$ and $\chi_{c 2}$ to the total number of $J / \psi$ for $T=160 \mathrm{MeV}$. This quantity, $R_{\chi_{c}}$ was measured by HERA-B collaboration in proton-nucleus collisions [66]. Our result shows $10-20 \%$ increase of this quantity for the statistical production with mass reductions, almost independent of the uncertain $\psi^{\prime}$ mass shift.

\section{SUMMARY}

In this paper, we investigate the mass shift of charmonia induced by change of gluon condensates in hadronic medium by making use of both perturbative (QCD second-order Stark effect) and nonperturbative (QCD sum rule) approaches. The inputs for the medium effect are the gluon condensates calculated by a resonance gas model that well reproduces the thermodynamic quantities calculated on the lattice QCD result in the temperature region considered here. Extending it to the finite baryonic chemical potential, we found that the change of the gluon condensates becomes larger at large chemical potential, which could result in a larger medium effect on charmonium production in heavy ion collisions at lower colliding energies, depending on hadronization temperature. We found that both the perturbative and nonperturbative estimations give almost the same mass shift. After elaborating on the sum rule analysis, we estimated systematic error on the obtained mass shift and found that most of the uncertainty comes mainly from thermal parameters. While the mass shift is almost constant along the chemical freeze-out line of which freeze-out temperature decreases as chemical potential increases (FOI), it can exhibit stronger downward shift at lower colliding energies if the charmonia are produced at hadronization and simultaneously frozen out (FOII).

We consider experimental implications of this observation in the context of statistical hadronization picture in which hadronization and the freeze-out of charmonium occur simultaneously. We found the data in $\mathrm{Pb}+\mathrm{Pb}$ collisions at $17 A \mathrm{GeV}$ are consistent with mass reduction of $J / \psi$ and $\chi_{c}$, although the effect on $\psi^{\prime}$ should be clarified before final conclusion is made. We pointed out that $10-20 \%$ enhancement of the production ratio between $\chi_{c}$ and $J / \psi$ could be a signature of the downward mass shift, which indicate a precursor of the deconfinement phenomenon.

\section{ACKNOWLEDGMENTS}

K.M. would like to thank K. Redlich and W. Weise for fruitful discussions during YIPQS international workshop on "New Frontiers in QCD 2010." This work was supported by Korean Ministry of Education through the BK21 Program and KRF-2011-0020333. K.M.'s work is supported by Frankfurt institute of Advanced Studies and the YIPQS program at Kyoto University. The work of S.H. was supported in part by the Asia Pacific Center for Theoretical Physics through the topical research program "New perspectives on sQGP."

\section{APPENDIX: DERIVATION OF THE SECOND-ORDER STARK EFFECT FORMULA FROM POTENTIAL NRQCD}

In this Appendix, we show that the second-order Stark effect, Eq. (13), can be derived from potential nonrelativistic QCD (pNRQCD) which provides a systematic perturbative approach to the OPE.

Here, the effective lagrangian in the static limit is given by $[67,68]$

$$
\begin{aligned}
\mathcal{L}= & -\frac{1}{4} G_{\mu \nu}^{a} G^{a \mu \nu}+\sum_{i=1}^{n_{f}} \bar{q}_{i} i D / q_{i} \\
& +\int d^{3} r \operatorname{Tr}\left\{\mathrm{S}^{\dagger}\left[i \partial_{0}+C_{F} \frac{\alpha_{V_{S}}}{r}\right] \mathrm{S}\right. \\
& \left.+\mathrm{O}^{\dagger}\left[i D_{0}-\frac{1}{2 N_{c}} \frac{\alpha_{V_{O}}}{r}\right] \mathrm{O}\right\}
\end{aligned}
$$




$$
\begin{aligned}
& +V_{A} \operatorname{Tr}\left\{\mathrm{O}^{\dagger} \vec{r} \cdot g \vec{E} \mathrm{~S}+\mathrm{S}^{\dagger} \vec{r} \cdot g \vec{E} \mathrm{O}\right\}+\frac{V_{B}}{2} \operatorname{Tr}\left\{\mathrm{O}^{\dagger} \vec{r} \cdot g \vec{E} \mathrm{O}\right. \\
& \left.+\mathrm{O}^{\dagger} \mathrm{O} \vec{r} \cdot g \vec{E}\right\}+\cdots .
\end{aligned}
$$

The fields $\mathrm{S}=S \mathbf{1}_{c} / \sqrt{N_{c}}$ and $\mathrm{O}=O^{a} T^{a} / \sqrt{T_{F}}$ are normalized static quark-antiquark singlet and octet fields, respectively, $\vec{E}$ is the chromoelectric field, and $C_{F}=\left(N_{c}^{2}-1\right) /\left(2 N_{c}\right)=4 / 3$. The trace is over the color indices. The matching coefficients at leading order are $\alpha_{V_{S}}=\alpha_{s}, \alpha_{V_{o}}=\alpha_{s}, V_{A}=1, V_{B}=1$. The leading-order correction to the singlet potential at finite temperature as given in Eq. (64) of Ref. [69] is

$$
\begin{aligned}
{\left[\delta V_{S}(r)\right]_{11}=} & -i g^{2} \frac{T_{F}}{N_{c}} \frac{r^{2}}{d-1} \int_{0}^{\infty} d t e^{-i t \Delta V} \\
& \times\left[\left\langle\vec{E}^{a}(t) \phi(t, 0)_{a b} \vec{E}^{b}(0)\right\rangle_{T}\right]_{11},
\end{aligned}
$$

where

$$
\Delta V=\frac{1}{r}\left(\frac{\alpha_{V_{o}}}{2 N_{c}}+C_{F} \alpha_{V_{S}}\right) \approx \frac{N_{c} \alpha_{s}}{2 r}
$$

and is the potential difference between the singlet ground state and the octet state excited by the color electric field. $d$ is the number of the dimension in the regularization of the momentum integral. In Ref. [69], the electric propagator is calculated in thermal perturbation in various limits.

To obtain the formula for the second-order Stark effect Eq. (A2), we have to take the following steps:

(i) First, to extract the contribution from the lowestdimensional operator, we take

$$
\left[\left\langle\vec{E}^{a}(t) \phi(t, 0)_{a b} \vec{E}^{b}(0)\right\rangle_{T}\right]_{11} \rightarrow\left[\left\langle\vec{E}^{a}(0) \vec{E}^{a}(0)\right\rangle_{T}\right]_{11} .
$$

Moreover, since we are interested in temperatures near $T_{c}$, instead of using thermal perturbation to calculate the temperature-dependent part of the electric condensate, we use the nonperturbative value extracted from lattice QCD. This approximation is valid as long as the scale in the matrix element is smaller than the separation scale.

(ii) We take the matrix element of Eq. (A2) for the groundstate charmonium and calculate it using the relative momentum between the $c \bar{c}$ quarks:

$$
\begin{aligned}
\delta E_{J / \psi}= & -i g^{2} \frac{T_{F}}{N_{c}} \frac{-i}{d-1} \int \frac{d^{3} p}{(2 \pi)^{3}} \frac{r^{2}}{E_{O}-E_{J / \psi}} \\
& \times|\psi(p)|^{2}\left\langle E^{a}(0) E^{a}(0)\right\rangle_{T} .
\end{aligned}
$$

(iii) We assume that the energies for the intermediate octet charmonium sate and the initial ground state can be written as follows:

$$
\begin{aligned}
E_{J / \psi} & =2 m_{c}-\epsilon \\
E_{O} & =2 m_{c}+p^{2} / m_{c},
\end{aligned}
$$

where $\epsilon$ is the binding energy for the $J / \psi$ and $E_{O}$ represents the energy of the octet continuum state. Putting these energies into Eq. (A5) and provided $T_{F}=1 / 2, d=4$, and $N_{c}=3$, one obtains Eq. (13). The contribution from the repulsive coulomb potential was neglected in the continuum octet energy as it vanishes in the large $N_{c}$ limit taken in the Peskin formalism [38].

The leading-order OPE term taken here is quite similar to the limit taken in Refs. [70,71]. The difference there was the assumption of Lorentz invariance of the vacuum, which is broken at finite temperature; therefore, the mass shift was proportional to the gluon condensate. As we have identified the approximations taken in the derivation of the second-order Stark effect, it would be useful to improve the formula by taking into account the renormalization group improved potentials and the $1 / N_{c}$ corrections [68].
[1] T. Matsui and H. Satz, Phys. Lett. B 178, 416 (1986).

[2] T. Hashimoto, O. Miyamura, K. Hirose, and T. Kanki, Phys. Rev. Lett. 57, 2123 (1986).

[3] E. Eichten, K. Gottfried, T. Kinoshita, K. D. Lane, and T. M. Yan, Phys. Rev. D 17, 3090 (1978).

[4] M. Asakawa and T. Hatsuda, Phys. Rev. Lett. 92, 012001 (2004).

[5] S. Datta, F. Karsch, P. Petreczky, and I. Wetzorke, Phys. Rev. D 69, 094507 (2004).

[6] T. Umeda, K. Nomura, and H. Matsufuru, Eur. Phys. J. C 39, 9 (2005).

[7] A. Jakovác, P. Petreczky, K. Petrov, and A. Velytsky, Phys. Rev. D 75, 014506 (2007).

[8] G. Aarts, C. Allton, M. B. Oktay, M. Peardon, and J.-I. Skullerud, Phys. Rev. D 76, 094513 (2007).

[9] For a recent review on potential models at finite temperature, see e.g. Á. Mócsy, Eur. Phys. J. C 61, 705 (2009).

[10] K. Morita and S. H. Lee, Phys. Rev. Lett. 100, 022301 (2008).

[11] K. Morita and S. H. Lee, Phys. Rev. C 77, 064904 (2008).

[12] Y. H. Song, S. H. Lee, and K. Morita, Phys. Rev. C 79, 014907 (2009).
[13] S. H. Lee and K. Morita, Phys. Rev. D 79, 011501 (2009).

[14] K. Morita and S. H. Lee, Phys. Rev. D 82, 054008 (2010).

[15] G. Boyd, J. Engles, F. Karsch, E. Laermann, C. Legeland, M. Lütgemeier, and B. Petersson, Nucl. Phys. B 469, 419 (1996).

[16] M. Panero, Phys. Rev. Lett. 103, 232001 (2009).

[17] S. Borsányi, G. Endrödi, Z. Fodor, A. Jakovác, S. D. Katz, S. Krieg, C. Ratti, and K. K. Szabó, JHEP 11 (2010) 077.

[18] Y. Aoki, G. Endrödi, Z. Fodor, S. D. Katz, and K. K. Szabó, Nature (London) 443, 675 (2006).

[19] S. Borsányi, Z. Fodor, C. Hoelbling, S. D. Katz, S. Krieg, C. Ratti, and K. K. Szabó, JHEP 09 (2010) 073.

[20] M. Cheng, N. H. Christ, S. Datta, J. van der Heide, C. Jung, F. Karsch, O. Kaczmarek, E. Laermann, R. D. Mawhinney, C. Miao et al., Phys. Rev. D 77, 014511 (2008).

[21] A. Bazavov et al. Phys. Rev. D 80, 014504 (2009).

[22] T. Hatsuda, Y. Koike, and S. H. Lee, Nucl. Phys. B 394, 221 (1993).

[23] J. Cleymans, H. Oeschler, K. Redlich, and S. Wheaton, Phys. Rev. C 73, 034905 (2006). 
[24] A. Andronic, P. Braun-Munzinger, and J. Stachel, Nucl. Phys. A 772, 167 (2006).

[25] A. Andronic, F. Beutler, P. Braun-Munzinger, K. Redlich, and J. Stachel, Phys. Lett. B 678, 350 (2009).

[26] F. Klingl, S. Kim, S. H. Lee, P. Morath, and W. Weise, Phys. Rev. Lett. 82, 3396 (1999).

[27] K. Nakamura et al., J. Phys. G.: Nucl. Part. Phys. 37, 075021 (2010).

[28] P. Koch, J. Rafelski, and W. Greiner, Phys. Lett. B 123, 151 (1983).

[29] S. Dürr, Z. Fodor, J. Frison, C. Hoelbling, R. Hoffmann, S. D. Katz, S. Krieg, T. Kurth, L. Lellouch, T. Lippert et al., Science 322, 1224 (2008).

[30] B. Borasoy and U. G. Meißner, Phys. Lett. B 365, 285 (1996).

[31] M. Glück, E. Reya, and A. Vogt, Z. Phys. C 53, 127 (1992).

[32] M. Glück, E. Reya, and I. Schienbein, Eur. Phys. J. C 10, 313 (1999).

[33] A. Bazavov and P. Petreczky, PoS (LATTICE2010), p. 169 (2010).

[34] A. Majumder and B. Müller, Phys. Rev. Lett. 105, 252002 (2010).

[35] D. H. Rischke, M. I. Gorenstein, H. Stöcker, and W. Greiner, Z. Phys. C 51, 485 (1991).

[36] M. M. Aggarwal et al. ( STAR Collaboration), Phys. Rev. C 83, 034910 (2011).

[37] O. Kaczmarek, F. Karsch, E. Laermann, C. Miao, S. Mukherjee, P. Petreczky, C. Schmidt, W. Soeldner, and W. Unger, Phys. Rev. D 83, 014504 (2011).

[38] M. E. Peskin, Nucl. Phys. B 156, 365 (1979).

[39] M. Luke, A. V. Manohar, and M. J. Savage, Phys. Lett. B 288, 355 (1992).

[40] S. H. Lee and C. M. Ko, Phys. Rev. C 67, 038202 (2003).

[41] A. Andronic, P. Braun-Munzinger, K. Redlich, and J. Stachel, Phys. Lett. B 659, 149 (2008).

[42] R. A. Bertlmann, Nucl. Phys. B 204, 387 (1982).

[43] R. J. Furnstahl, T. Hatsuda, and S. H. Lee, Phys. Rev. D 42, 1744 (1990).

[44] O. Kaczmarek and F. Zantow, Phys. Rev. D 71, 114510 (2005).

[45] L. J. Reinders, H. Rubinstein, and S. Yazaki, Phys. Rep. 127, 1 (1985).

[46] B. L. Ioffe, Prog. Part. Nucl. Phys. 56, 232 (2006).
[47] M. A. Shifman, A. I. Vainshtein, and V. I. Zakharov, Nucl. Phys. B 147, 385 (1979).

[48] A. I. Bochekarev and M. E. Shaposhnikov, Nucl. Phys. B 268, 220 (1986).

[49] G. Aarts and M. M. Resco, Nucl. Phys. B 726, 93 (2005).

[50] Á. Mócsy and P. Petreczky, Phys. Rev. D 73, 074007 (2006).

[51] T. Umeda, Phys. Rev. D 75, 094502 (2007).

[52] Y. Koike, Phys. Rev. C 51, 1488 (1995).

[53] T. Hatsuda, S. H. Lee, and H. Shiomi, Phys. Rev. C 52, 3364 (1995).

[54] S. Kim and S. H. Lee, Nucl. Phys. A 679, 517 (2001).

[55] R. Rapp, D. Blaschke, and P. Crochet, Prog. Part. Nucl. Phys. 65, 209 (2010).

[56] X. Zhao and R. Rapp, Phys. Lett. B 664, 253 (2008).

[57] A. Mócsy and P. Petreczky, Phys. Rev. D 77, 014501 (2008).

[58] Y. Park, K. I. Kim, T. Song, S. H. Lee, and C. Y. Wong, Phys. Rev. C 76, 044907 (2007).

[59] T. Song, W. Park, and S. H. Lee, Phys. Rev. C 81, 034914 (2010).

[60] M. Gaździcki and M. I. Gorenstein, Phys. Rev. Lett. 83, 4009 (1999).

[61] A. Andronic, P. Braun-Munzinger, K. Redlich, and J. Stachel, Phys. Lett. B 571, 36 (2003).

[62] A. Andronic, P. Braun-Munzinger, K. Redlich, and J. Stachel, Phys. Lett. B 652, 259 (2007).

[63] F. Karsch, D. Kharzeev, and H. Satz, Phys. Lett. B 637, 75 (2006).

[64] B. Alessandro et al. (NA50 Collaboration), Eur. Phys. J. C 39, 335 (2005).

[65] L. Grandchamp and R. Rapp, Nucl. Phys. A 709, 415 (2002).

[66] I. Abt et al. (HERA-B Collaboration), Phys. Rev. D 79, 012001 (2009).

[67] N. Brambilla, A. Pineda, J. Soto, and A. Vairo, Nucl. Phys. B 566, 275 (2000).

[68] N. Brambilla, A. Pineda, J. Soto, and A. Vairo, Rev. Mod. Phys. 77, 1423 (2005).

[69] N. Brambilla, J. Ghiglieri, A. Vairo, and P. Petreczky, Phys. Rev. D 78, 014017 (2008).

[70] M. B. Voloshin, Nucl. Phys. B 154, 365 (1979).

[71] H. Leutwyler, Phys. Lett. B 98, 447 (1981). 